\title{
N94-10601
}

\section{NEW SQUEEZED LANDAU STATES}

\author{
C. Aragone \\ Departamento de Física, Univ. S. Bolivar \\ Apartado 89000, Caracas $1080 \mathrm{~A}$
}

\begin{abstract}
A bstract
We introduce a new set of squeezed sates through the coupled two-mode squeezed operator. It is shown their behaviour is simpler than the correlated coherent states introduced by Dodonov, Kurmyshev and Man'ko in order to quantum mechanically describe the Landau system, i.e. a planar charged particle in a uniform magnetic field. We compare results for both sets of squeezed states.
\end{abstract}

A planar charged particle moving in a uniforn magnetic field is a very interesting quantum mechanical system. It is not trivial, needs the two spatial dimensions to describe it, it has some reminiscence of the two dimensional oscillator, but requires in addition the peculiar presence of the angular momentum operator which play a role as important as the hamiltonian. As recently it has been pointed out [1], the system has an Osc(1) dynamical degeneracy group. It seemed to us the system has a physics rich enough and mathematically particularly well understood in terms of the holomorphic (and antiholomorphic) coordinates that deserved to be revisited.

$\Lambda$ planar particle of charge $e$, mass $m$, moving in a uniform magnetic field $\vec{B}=B \hat{k}$ can be described by the classical first order action

$$
S=\left\langle\vec{p} \cdot \dot{\vec{r}}-(2 m)^{-1}\left[\vec{p}-2^{-1} e B(i \vec{r})\right]^{2}\right\rangle \sqsubseteq\langle\vec{p} \cdot \dot{\vec{r}}-H\rangle .
$$

$\vec{r}$ is the two-dimensional vector position of $e, \vec{p}$ its canonical momenta (which in the presence of the vector potential $\vec{A}=2^{-1} B(i \vec{r})$ does not coincide with $m \dot{\vec{r}}$ ), and the linear operator $i$ indicates a positive $\pi / 2$ rotation, i.e. $(i \vec{v})_{j}=-\epsilon_{j l} v_{l}$. We choose $B$ such that $e B \leftrightarrows m \omega$ is always positive, without losing generality.

The Landau system $\Phi_{L} \rightrightarrows\{\vec{r}, \vec{p}, H, \Lambda \equiv-(i \vec{r}) \cdot \vec{p}\}$ is quantized by imposing

$$
\left[r_{i}, p_{j}\right]=i \hbar \delta_{i j} \quad i, j=(1,2) \text {. }
$$

As shown in ref. [1] it is convenient to introduce two sets of additional, momentum-like variables

$$
\vec{\pi} \equiv \vec{p}-2^{-1} m \omega(i \vec{r}), \quad \vec{\omega} \equiv \vec{p}+2^{-1} m \omega(i \vec{r}) .
$$

$\vec{\pi}$ is the q-operator representing the observable $m \dot{\vec{r}}$. In terms of these quantities the hamiltonian and the angular momentum take the form

$$
H=(2 m)^{-1}\left\{\vec{p}^{2}+4^{-1} m^{2} \omega^{2} \vec{r}^{2}+m \omega \Lambda\right\}=(2 m)^{-1} \vec{\pi}^{2},
$$




$$
\Lambda=(-i \vec{r}) \cdot \vec{p}=(2 m \omega)^{-1}\left\{\vec{\pi}^{2}-\vec{\omega}^{2}\right\} .
$$

Observe the irteresting chiral aspect of $\Lambda$ in terms of $\vec{\pi}$ and $\vec{\omega}$.

It is inmediate to notice that $\omega_{i}$ commutes with $\pi_{j}$,

$$
\left[\omega_{i}, \pi_{j}\right]=0
$$

Consequently $\vec{\omega}$ and $\Lambda$ commute with $H$. Since

$$
\begin{gathered}
{\left[\Lambda, \omega_{i}\right]=-i \hbar \epsilon_{i j} \omega_{j}=i \hbar(i \vec{\omega})_{i}} \\
{\left[\omega_{i}, \omega_{j}\right]=-i \hbar m \omega \epsilon_{i j} .}
\end{gathered}
$$

we see that $\{1, \vec{w}, \Lambda\}$ constitute a dynamical symmetric group (which will be easily recognized, when represented by its holomorphic components $\omega_{z}, \omega_{z}$ to be Osc (1)), i.e. commutes with $H$.

It is convenient to introduce holomorphic dimensionless variables $z, \bar{z}, p_{z}, p_{\bar{z}}, \pi_{z}, \pi_{\bar{z}}, \omega_{z}, \omega_{\bar{z}}$ to analyze the system,

$$
z \equiv\left(2^{-1} \hbar^{-1} m \omega\right)^{1 / 2}(x+i y), \quad p_{z}=(2 \hbar m \omega)^{1 / 2}\left(p_{x}-i p_{y}\right)=-i \partial_{z}+c . c .
$$

The two momentum-like set of variables take the form

$$
\begin{array}{ll}
\pi_{z}=p_{z}+2^{-1} i \bar{z}, & \pi_{\bar{z}}=p_{z}-2^{-1} i z \\
\omega_{z}=p_{z}-2^{-1} i \bar{z}, & \omega_{z}=p_{z}+2^{-1} i z
\end{array}
$$

while $I I$ and $\Lambda$ become

$$
\begin{gathered}
H=\hbar \omega\left\{p_{x} p_{\bar{z}}+4^{-1} z \bar{z}+2^{-1} \lambda\right\} \leftrightarrows \hbar \omega h, \\
\Lambda=i \hbar\left\{\bar{z} p_{\bar{z}}-z p_{z}\right\} \sqsubseteq \hbar \lambda=\hbar\left[\bar{z} \partial_{\bar{z}}-z \partial_{z}\right] .
\end{gathered}
$$

Ileinsenberg commutation relations eqs. (2) change to

$$
\left[z, p_{2}\right]=i=\left[z, \pi_{2}\right]=\left[z, \omega_{2}\right]+\text { c.c. . }
$$

The two main physical observables $h, \lambda$ have a very simple structure

$$
h=\pi_{x} \pi_{\overline{7}}+2^{-1} \sqsubseteq n_{1}+2^{-1}, \lambda=\pi_{2} \pi_{\bar{z}}-\omega_{\bar{z}} \omega_{2} \sqsubseteq n_{1}-n_{2}
$$

where $\pi_{\bar{z}}, \omega_{z}, \pi_{z}, \omega_{\bar{z}}$ can be regarded as two sets of decoupled annihilation and creation operators

$$
\left[\pi_{\bar{z}}, \pi_{z}\right]=1=\left[\omega_{z} \omega_{\bar{z}}\right] \text {, }
$$

since $\left[\omega_{z, \bar{z}}, \pi_{z, \bar{z}}\right]=0$. We emphasize the fundamental role of the both $h, \lambda(H, \Lambda)$ in determining the two-mode quantum structure of the system, The energy degeneracy is broken by the presence of $n_{2}$, the second fundamental quantum number. These two series of discretes numbers will become the origin of the two couplex parameters labelling the coherent Landau states dicovered long time 
ago [2] by Mal'kin and Man'ko. (Incidentally our $\pi_{\bar{z}}$ coincides with $a$ of ref. [3] and our $\omega_{z}$ equals $-i a_{0}$. To introduce coherent Landau states we introduce the state $\mid 0,0>=\psi_{00}$

$$
\psi_{00}(z \bar{z})=\pi^{-\frac{1}{2}} e^{-\frac{1}{2} z \bar{z}}
$$

$\psi_{00}$ belongs to the ground subspace, i.e. $\pi_{F} \mid 0,0>=0$ and is unitary (using the natural measure $2^{-1} i d z d \bar{z}=d x d y$. The ground subspace is determined by the orthonormal set $\psi_{0 p}=(p !)^{-1 / 2}$ $\left.\omega_{\frac{p}{z}}^{p}|0,0>=| 0, p\right\rangle$

$$
\left|0, p>=(p !)^{-\frac{1}{2}}(i z)^{p}\right| 0,0>\text {. }
$$

Each level- $n$ energy eigenspace has the discrete orthonormal basis

$$
\psi_{n p}=(n !)^{-\frac{1}{2}}(p !)^{-\frac{1}{2}} \pi_{z}^{n} \omega_{\frac{p}{z}}^{p} \mid 0,0>.
$$

Equations (14) tell us $H \psi_{n p}=\hbar \omega\left(n+2^{-1}\right) \psi_{n p}$ and $\Lambda \psi_{n p}=\hbar(n-p)$.

We define the coherent Landau states [2] by

$$
\left|w, s>\equiv e^{w \pi_{2}-w \pi_{T}+w_{T}-\pi w_{2}}\right| 0,0>
$$

$w, s \in \mathscr{A}$. They constitute an over complete unitary system of the Hilbert space $\left\{\psi_{n p}, n ; p \in\right.$ $0,1, \cdots\}$ in the usual sense (for coherent states)

$$
\left\langle w_{1} s_{1} \mid w_{2} s_{2}\right\rangle=e^{-\frac{1}{2}\left|w_{2}-w_{1}\right|^{2}-\left|\varphi_{2}-s_{1}\right|^{2}+i\left|w_{2}\right|\left|w_{1}\right| \sin \left(\varphi_{2}-\varphi_{1}\right)+i\left|s_{2}\right|\left|s_{1}\right| \sin \left(\phi_{3}-\phi_{1}\right)}
$$

$w=|w| e^{i \varphi}, s=|s| e^{i \phi}$.

They have three basic properties: $i$. They are $\pi_{z}$ eigenstates with eigenvalue $w$, ii. they also are eigenstates of $\omega_{z}$ with proper value $s$

$$
\pi_{\bar{z}}|\mathrm{w} s>=\mathrm{w}| \mathrm{w}, s>, \omega_{z}|w s>=s| \mathrm{w} s>,
$$

and iii. they propagate remaining in the family. If one starts on $\mid w s>$ leaving the system to evolve, at time $t \Phi_{L}$ will be described by

$$
e^{-i h \omega t}|w s>=| w e^{-i \omega t}, s>\text {. }
$$

Eqs. (20) suggest a way to compute q-mechanical expected values for physical observables $F(p, \bar{p}, z, \bar{z})$. One has to transform them lo their representation in terms of the new variables $(\pi, \bar{\pi}, \omega, \bar{\omega})$, then normal ordering in both types of variables and finally taking into account eqs. (20).

In this way we obtain:

$$
\begin{gathered}
<z>_{C L}=<\mathrm{w} s|z| \mathrm{w} s>=<\mathrm{w} s\left|\left(i \pi_{\bar{s}}-i \omega_{\bar{s}}\right)\right| \mathrm{w}, s>=i(\mathrm{w}-\bar{s}) \\
<z^{2}>_{C L}=-(\mathrm{w}-\bar{s})^{2},\langle z \bar{z}>=(\mathrm{w}-\bar{s})(\bar{w}-s)+1
\end{gathered}
$$

plus their respective complex (hermitian) conjugates. We also obtain

$$
<p_{z}>_{C L}=2^{-1}<\pi_{z}+\omega_{z}>_{C L}=2^{-1}(\bar{w}+s)+\text { c.c. }
$$




$$
\begin{gathered}
<p_{z}^{2}>_{C L}=4^{-1}<\left(\pi_{z}+w_{z}\right)^{2}>=4^{-1}(\bar{w}+s)^{2}+h . c . \\
<p_{z} p_{\bar{z}}>_{C L}=4^{-1}+4^{-1}(w+\bar{s})(\bar{w}+s) \\
<h>_{C L}=<w s|h| w s>=w \bar{w}+2^{-1}, \quad<\lambda>_{C L}=w \bar{w}-s \bar{s}, \\
<h^{2}>_{C L}=\left(w \bar{w}+2^{-1}\right)^{2}+w \bar{w}, \quad<\lambda^{2}>_{C L}=<\lambda>_{C L}^{2}+w \bar{w}+s \bar{s} .
\end{gathered}
$$

Recalling definitions (8) relating $z, \bar{z}$ and real dimensionless variables $x, y$ we can calculate physical uncertanties, which are defined for canonical sets of variables in terms of holomorphic variances $\Delta z, \Delta z \bar{z}=\langle z \bar{z}\rangle-\langle z\rangle\langle\bar{z}\rangle, \Delta p_{z}, \Delta p_{z} p_{\bar{z}}$. they turn out to be

$$
(\Delta x)_{C L}^{2}=4^{-1}(\Delta z)_{C L}^{2}+4^{-1}(\Delta \bar{z})_{C L}^{2}+2^{-1}(\Delta z \bar{z})_{C L}=2^{-1}=(\Delta y)_{C L}^{2},(\Delta x y)_{C L}=0 .
$$

In a similar way, we find for the physical momenta

$$
\left(\Delta p_{x}\right)_{C L}^{2}=2^{-1}=\left(\Delta p_{x} p_{y}\right)_{C L}=0 .
$$

Consequently both uncertanties attain lowest bound

$$
(\Delta x)_{C L}\left(\Delta p_{x}\right)_{C L}=2^{-1}=(\Delta y)_{C L}\left(\Delta p_{y}\right)_{C L}
$$

Coherent Landau states are minimun uncertanty states (MUS).

Squeezing can be now analysed, since the standard procedure to consider this type of states involves the squeezing of associated coherents states. Complexive decoupled squeezed Landau states have been introduced in ref. [3], where they have been called correlated coherent states.

Since squeezing is not that intuitive we face in principle four different types of squeezing: partial squeezing in $\pi_{\bar{z}} \pi_{z}$, partial squeezing in $\omega_{z} \omega_{\bar{z}}$ or full, complexive squeezing in both sets of variables.

The complexive squeezing might be either decoupled or coupled in both set of variables. One might think that it could be enough to squeeze just in the dynamical constituents of the hamiltonian $\pi_{\bar{z}} \pi_{z}$ in order to oblain physically appealing results. This primary type of "squeezing" can be shown to lead to states which are irrelevant, since they are neither minimun uncertainty states nor the variances of any canonical variable can tend to zero.

We are obliged to turn our interest to more radical way of squeezing. As we said above, we must try complexive squeezing, i.e. to introduce squeeze operators which squeeze both type of quanla, the $\pi$ and the $\omega$-ones.

Let us first consider what we call "decoupled" squeezing, as it has been done in ref. [3]. The squeezing operator is defined as

$$
S\left(q_{1}, q_{2}\right)=e^{\frac{1}{2} q_{1}^{2} \pi_{2}^{2}-\frac{1}{2} \bar{q}_{1}^{2} \pi_{\frac{2}{2}}^{2}+\frac{1}{2} q_{2}^{2} \omega_{2}^{2}-\frac{1}{2} \bar{q}_{2}^{2} \omega_{2}^{2}}=S^{\pi}\left(q_{1}\right) S^{\omega}\left(q_{2}\right) .
$$

We consider the squeezed states

$$
\left|w s, q_{1}, q_{2}\right\rangle \equiv S\left(q_{1}, q_{2}\right)|w, s\rangle \text {. }
$$

where both $w$ and $s$ are distorted. 
Both the $\pi$ and $\omega$ variables transform non trivially here,

$$
\begin{gathered}
\left(\pi_{\bar{z}}\right)_{q_{1}} \rightrightarrows S_{q_{1}}^{+} \pi_{\bar{z}} S_{q_{1}}=\pi_{\bar{z}} c h r_{1}+e^{2 i \varphi_{1}} s h r_{1} \pi_{z} \\
\left(\omega_{\bar{z}}\right)_{q_{2}} \rightrightarrows S_{q_{2}}^{+} \omega_{z} S_{q_{2}}=\omega_{z} c h r_{2}+e^{2 i \varphi_{2}} s h r_{2} \omega_{\bar{z}} . \quad+h . c .
\end{gathered}
$$

The squcezed transformed of the Heinserberg canonical variable $z z_{q 192} \equiv S_{q 192}^{+} z S_{q 192}$ becomes in the present case

$$
z_{q_{1} q_{2}}=i\left(\pi_{\bar{z}} c h r_{1}+e^{2 i \varphi_{1}} s h r_{1} \pi_{z}-\omega_{\bar{z}} c h r_{2}-e^{-2 i \varphi_{2}} s h r_{2} \omega_{2}\right) .
$$

The complexive squeezed expectation values of $z$ and $p_{z}$ are therefore

$$
\begin{gathered}
<z>_{q 1 q 2}=<z_{q 1 q 2}>_{C L}=i\left[s_{q 1}(w)-\bar{s}_{q 2}(s)\right], \\
<p_{z}>_{q 1 q 2}=<\left(p_{z}\right)_{q 1 q 2}>_{C L}=2^{-1}\left[\bar{s}_{q 1}(w)+s_{q 2}(s)\right],
\end{gathered}
$$

where subindex $C L$ indicates the coherent Landau state $\mid w, s>$ and $s_{q}(u) \equiv c h r u+e^{2 i \varphi} s h r \bar{u}$. Quadratic complexive squcezed expectation values become

$$
\begin{gathered}
<z^{2}>_{q_{1} q_{2}}=-\left[s_{q_{1}}(w)-\bar{s}_{q_{2}}(s)\right]^{2}-s h r_{1} c h r_{1} e^{2 i \varphi_{1}}-s h r_{2} c h r_{2} e^{-2 i \varphi_{2}} \\
<z \bar{z}>_{q_{1} q_{2}}=<\bar{z}>_{q 1 q_{2}}<z>_{q_{1} q_{2}}+c h r_{1}^{2}+s h r_{2}^{2}, \\
<p_{z}^{2}>_{q_{1} q_{2}}=4^{-1}\left(\bar{s}_{q_{1}}(w)+s_{q_{2}}(s)\right)^{2}+4^{-1} s h r_{1} c h r_{1} e^{-2 i \varphi_{1}}+4^{-1} s h r_{2} c h r_{2} e^{2 i \varphi_{2}}, \\
<p_{z} p_{\bar{z}}>_{q_{1} q_{2}}=4^{-1}\left[\bar{s}_{q_{1}}(w)+s_{q_{2}}(s)\right]\left[s_{q_{1}}(w)+\bar{s}_{q_{2}}(s)\right] 4^{-1} \operatorname{ch}^{2} r_{1}+4^{-1} s h^{2} r_{2} .
\end{gathered}
$$

From this expressions for the holomorphic variables we can evaluate physical uncertanties to see how they behave for complexive decoupled squeezing. They are

$$
\begin{aligned}
\left(\Delta p_{y}\right)_{q 1 q_{2}}^{2}= & (\Delta x)_{q_{192}}^{2}=2^{-1} \operatorname{ch} r_{1}\left(\operatorname{ch} r_{1}-s h r_{1} \cos 2 \varphi_{1}\right)+2^{-1} \operatorname{ch} r_{2}\left(\operatorname{ch} r_{2}-s h r_{2} \cos 2 \varphi_{2}\right)-2^{-1} \\
(\Delta y)_{q_{1} q_{2}}^{2}= & \left(\Delta p_{x}\right)_{q_{1} q_{2}}^{2}=2^{-1} \operatorname{ch} r_{1}\left(\operatorname{ch} r_{1}+s h r_{1} \cos 2 \varphi_{1}\right)+2^{-1} \operatorname{ch} r_{2}\left(\operatorname{ch} r_{2}+s h r_{2} \cos 2 \varphi_{2}\right)-2^{-1} \\
\text { For } \varphi_{1}= & 0=\varphi_{2} \quad \Delta x \text { and } \Delta p_{y} \text { are squeezed since: } \\
& \left(\Delta p_{y}\right)_{\varphi_{1}=0=\varphi_{2}}^{2}=(\Delta x)_{\varphi_{1}=0=\varphi_{2}}^{2}=4^{-1} e^{-2 r_{1}}+4^{-1} e^{-2 r_{2}} \rightarrow 0^{+}, r_{1}, r_{2} \rightarrow \infty
\end{aligned}
$$

while, of course $\Delta p_{x}$ and $\Delta y$ incrcase according to eq. (38). The partial uncertanties get closer to their lowest bound,

$$
(\Delta y)_{q_{1} q_{2}}^{2}=\left.\left(\Delta p_{x}\right)_{q_{1} q_{2}}^{2}\right|_{\varphi_{1}=0=\varphi_{2}}=8^{-1}\left[1+c h r_{2}\left(r_{2}-r_{1}\right)\right]=(\Delta y)_{q_{1} q_{2}}^{2}=\left.\left(\Delta p_{y}\right)_{q_{1} q_{2}}^{2}\right|_{\varphi_{1}=0=\varphi_{2}}
$$

This result indicates that physical squeezing, in the sense that the squeezed states are also minimun uncertanty states, is obtained just for $r_{2}=r_{1}$. Complexive decoupled squeezing leads to physical squcezing modes, but the two indepcndent "a priori" parameters $q_{1}$ and $q_{2}$ have to coincide.

A nicer solution to finding squeezed states of $\Phi_{l}$ arises by considering the fact that we have two modes in the system. For this situation a more natural squeezed operator can be defined, 
similarly to what has been done for the two photon case in ref. [4]. The "coupled" squeezing operator we postulate is given by

$$
S_{q} \equiv e^{\frac{1}{2} q^{2} x_{2} w_{\mathbf{T}}-\frac{1}{2} \bar{q}^{2} \bar{\pi}_{s} \omega_{\ell}} .
$$

It naturally depends upon only one parameter. It is straighforward to show that the squeezed yalues of $\pi_{\bar{z}}$ and $\omega_{z}$ respectively are

$$
\begin{aligned}
& S_{q}^{+} \pi_{\bar{z}} S_{q}=\pi_{\bar{z}} \operatorname{ch} \frac{r}{2}+e^{2 i \varphi} \operatorname{sh} \frac{r}{2} \omega_{\bar{z}}, \\
& S_{q}^{+} \omega_{x} S_{q}=\omega_{z} \operatorname{ch} \frac{r}{2}+e^{2 i \varphi} \operatorname{sh} \frac{r}{2} \pi_{z} .
\end{aligned}
$$

As expected this type of squeezing makes $\pi$-variables to have $\boldsymbol{\omega}$-components and viceversa.

The new associates squeezed states are defined by

$$
\left|\mathrm{w} s, q>\equiv S_{\vartheta}\right| \mathrm{w}, s>
$$

where $S_{q}$ has been introduced in eq. (41), It is inmediate to perform in this case similar calculations to what has already been done for the previous case. Results turn out to be mathematically simpler and physically interesting. We get

$$
\begin{gathered}
<z>_{q}=<z_{q}>_{C L}=i(w-\bar{s}) \operatorname{ch} \frac{r}{2}+i s h \frac{r}{2}\left(3 e^{2 i \varphi}-w e^{-2 i \varphi}\right), \\
<p_{z}>_{q}=2^{-1}(\bar{w}+s) \operatorname{ch} \frac{r}{2}+2^{-1} \operatorname{sh} \frac{r}{2}\left(\bar{w} e^{2 i \varphi}+s e^{-2 i \varphi}\right) .
\end{gathered}
$$

In addition one finds that $\left(\Delta_{z}\right)_{q}^{2}=\left(\Delta_{p_{s}}\right)_{q}^{2}=0$. Finally the variances of the canonical variables altain the respective forms.

$$
\begin{aligned}
& (\Delta x)_{q}^{2}=4^{-1} e^{r}(1-\cos 2 \varphi)+4^{-1} e^{-r}(1+\cos 2 \varphi)=\left(\Delta p_{y}\right)_{q}^{2} \\
& \left(\Delta p_{x}\right)_{q}^{2}=4^{-1} e^{r}(1+\cos 2 \varphi)+4^{-1} e^{-r}(1-\cos 2 \varphi)=(\Delta y)_{q}^{2}
\end{aligned}
$$

Both uncertanties coincide, their value being

$$
(\Delta x)_{q}^{2}\left(\Delta p_{x}\right)_{q}^{2}=(\Delta y)_{q}^{2}\left(\Delta p_{y}\right)_{q}^{2}=4^{-1}\left(c h r^{2}-s h r^{2} \cos ^{2} 2 \varphi\right) \text {. }
$$

For $\varphi=k \pi / 2$ we obtain squeezing and minimun uncertainty.

In conclusion we feel these coupled squeezed states (44) are the natural ones for introducing squeezing in the Landau system. We have shown they behave in a simpler way then those defined in ref. [3] while they also lead to physical squeezing.

\section{References}

[1] S. Fubini, International J. of Mod. Phys. As (1990) 3533.

[2] I. A. Mal'kin and V. I. Man'ko, ZhETF 55 (1968) 1014.

[3] V. V. Dodonov, E. V. Kurmyshev and V. I. Man'ko, Proc. Lebedev Physics Institute, Nova Science, Commack, N.Y. (1988) 176 pp 169.

(4) B. L. Schumaker and C. M. Caves, Phys. Rev. A31 (1985) 3093. 